\title{
Retina Cancer Detection using Thresholding Approach
}

\author{
Dr. Harsh S Dave ${ }^{1}$, Dr. Vaishnavi Patel ${ }^{2}$, Dr. Aash Gopalak ${ }^{3}$, Dr. Harsh Bhatt ${ }^{4}$, Dr. Sheshang Degadwala ${ }^{5}$ Dhairya Vyas ${ }^{6}$ \\ 1,3,4 MBBS, SBKS MI \& RC, India \\ ${ }^{2} \mathrm{MD}$, University of Perpetual Help Systems, Phillipines \\ ${ }^{5}$ Associate Professor, Sigma Institute of Engineering, Vadodara, Gujarat, India \\ ${ }^{6}$ Managing Director, Shree Drashti Infotech LLP, Vadodara, Gujarat, India
}

\section{Article Info}

Volume 8, Issue 1

Page Number: 276-282

Publication Issue :

January-February-2021

\section{Article History}

Accepted : 01 Jan 2021

Published : 28 Jan 2021

\section{ABSTRACT}

Giant biomedical robots including Lasik machine are used to detect cancer. Picture segmentation is an effective form of image analysis for retinal eye detection. A picture interpretation method for the diagnosis of eye cancer is established in this article. In this scheme, the cancer is divided into various image processing methods and marked on the original image. The pictures are smoothed with two dimensional filters. In order to apply the picture to the original, the backdrop is retracted and results in a better field of interest or region of cancer. The tests have shown that the device indicated is able to detect retina cancer in the eye based on an image threshold. The study suggested comprises of two naming stages, the Eye Detection System and the Smart Retina Cancer Detection System. In the search for eye cancer the results are compared accordingly.

Keywords : 2-D Filter, Morphology Operation, Hough Transform, Logical Operation and Thresholding

\section{INTRODUCTION}

Secondary glaucoma can be hard to detect with the retina disease. However, the right diagnosis and management can be done in almost all cases by the retention of a suitable index of suspicion and an ophthalmic exam through sufficient use of auxiliary tests. Cancer can be favoured (not harmful) cancer in the retina and in the tissue (all of which are known as the cycle), but can also threaten tumours like rhabdomyosarcoma and retina blastoma, for instance.
The retina is arranged as follows: the white portion of the sclera. The iris: the dark piece of the body that sweeps the student in the iris. Retina growth can be defined as an anomalous cell revolutionary production. This can be produced in or around the retina and then in a mass (cancer).

A cancer is an unregulated cell growth in a mass of tissues. There are many types of retinal cancer and certain areas of the eye may be influenced by each kind. The retina can be named the therapeutic name of the retina as a visual medicine. The interior of the eye or extra-ocular may be inside the eye, implying that it affects the outer part of the eye. Cyst, Nevus, 
and Melanoma are the most commonly accepted forms of intraocular iris tumours.

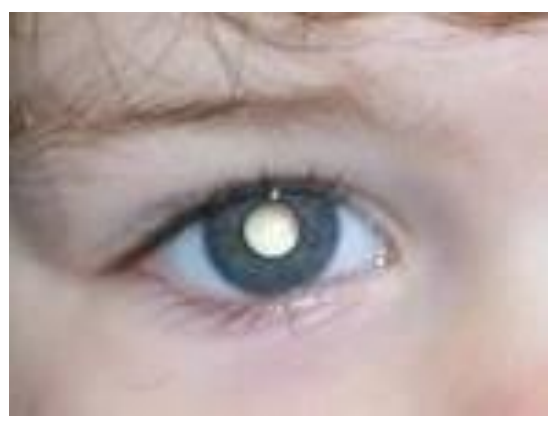

Figure 1: Cancer Retina

The development is a benevolent tumour inside the stroma, the iris front or the iris colour epithet; the iris again. Nevus is a kind of heart-like pigmented iris tumour, emphasised in the iris as yellow spots. It may be beneath or near the iris. Nevus has an unusual damage to facial melanoma; an eye cancer the retina is arranged as follows: the white portion of the sclera. The iris: the dark piece of the body that sweeps the student in the iris. Retina growth can be defined as an anomalous cell revolutionary production. It can be generated in or around the retina and then a mass (cancer).

Image manipulation methods are widely used in different fields of medicine. Photo compression is one of the most frequent diagnostic image treatment applications. In the medical industry, this application is critical as file size reduction enables more images to be saved in a particular volume of disc or memory space. To produce the best possible and most precise performance, image-processing approaches have been employed with the help of artificial intelligence technologies, such as the neural backbone network.

The objective of the paper is to develop a comprehensive method to identify the various iris tumours using image processing techniques. Any image of the provided knee must then be processed, and the tumour segmented using Hough circular detection algorithm, so that the original image can be marked. The technology suggested uses iris pictures from a public online database.

\section{RELATED WORKS}

In [1] develops an Eye Tumour Detection System (ETDS) as well as an IETDs system. The method proposed is an image processing paradigm combining various image processing techniques20 and two phased neural network back propagation. EDTS's aim is to identify tumours in the iris and to label them using image fusion on an original picture, while IEDTS detects tumours in the smart device. The programming language Matlab is used to incorporate and simulate the proposed framework (Matlab 2013a).

In [2] an image improvement tumour method is given. In [2]. To minimise analytical and timeconsuming data, imaging techniques are used to provide meaningful representations of lung patterns. In order to detect tumours inside images, relationships between the input and the output patterns were established. Image images are produced in a raw format $256 \times 256$, which is used for clearing black and white pixels by thresholds. Erosion and medium filtering were then used to eliminate noise. Small objects are then extracted inside the image.

In [4] design and installation of the method presented here uses a database of $120 \mathrm{x}$-ray images. Both methods of compression (DCT and HWT) were implemented with nine compression ratios for $\mathrm{x}$-ray images. On the basis of visual inspection and objective study, the optimum compression ratios of DCT and HWT for x-ray images is measured using optimum compression parameters. In order to 
equate the optimal compression ratios with the imaging entropy, linear regression analysis is applied as a mathematical approach. The neural network contrasts the resolution of $x$-ray images (pixel values) with the optimal compression of the images.

In [8] clinical and pathologic results are analysed and the similarities between histopathology and forecasts of iris melanoma, which vary greatly from choroidal melanoma, are highlighted. A higher metastatic rate than tumours, which consist of only one of the two iris melanoma (containing spindle $\mathrm{B}$ cells as well as epithelial cells) carries a mixed cell pathology. This compares with choroidal melanoma, which is the best pathologic predictor for inaccurate predictions with epithelioid cells. The eye result, which can be obtained with a well-delimited iris melanoma, without local operative excision.

In [11] method for detecting the iris tumour using segmentation and image fusion was proposed in this article. The method created reads, processes and labels the tumour iris image (tumour regions) including the pupil of the affected images. However, only students are labelled with natural photographs since the iris does not have tumours. The system was checked for standard and irregular images taken from Miles Research and effective trial findings proving that our efficient system can be used in real life applications.

There are two phases in [15] proposed NASM filter. To distinguish each pixel as unwrought pixels, insulated pulse noise, non-insulated impulses noise, or image objects' edge pixels, a soft-switching noise detection scheme is designed. The following characteristic form is identified: "Not filter" (or identity filter), the regular median filter (SM) or our Fuzzy weighted median (FWM) filter. Experimental findings reveal that our NASM filter outputs more techniques impressively across a reasonably narrow spectrum of noise densities, from $10 \%$ to $70 \%$, according to the ideal switching median filter.

\section{PROPOSED SYSTEM}

As shown in below figure 2 proposed system for retina cancer detection are perform in the flow.

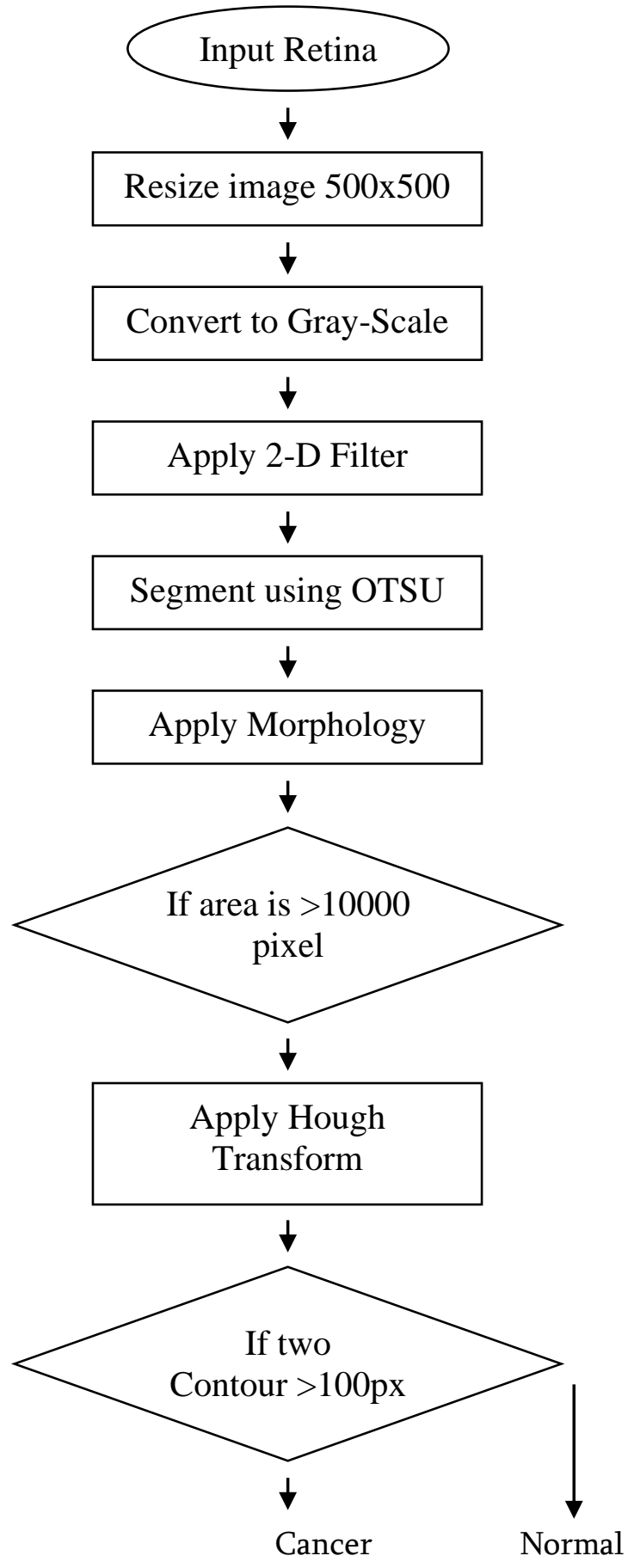

Figure 2: Proposed System Flow Diagram 


\section{A. Datasets [1]:}

Two related databases, "MilesResearch," and "EyeCancer" provide photographs for the analysis of the created framework. Both databases are open to the public on the internet. The photographs obtained would be transformed into grayscale for image processing.

The pictures are $500^{*} 500$ images in dimension. The first is collected with 25 images and the second is collected with 25 images. The photographs in the archive captured are split into normal and irregular photos. A total of 50 pictures are half grouped.

\section{B. Segmentation [5]:}

i.OTUS Thresholding [6]: Otsu method is one of the most successful methods for image thresholding. Converting a greyscale image to monochrome is a common image pro- cessing task. Otsu's method, named after its inventor Nobuyuki Otsu, is one of many binarization algorithms. In the simplest form, the algorithm returns a single intensity threshold that separate pixels into two classes, foreground and background.

ii.Morphological based segmentation [4]: Converting the images to binary reduces computational complexity and storage issues and also is a prerequisite for morphological segmentation of lungs.

- Morphological open operation,

$$
A \circ B=(A \theta B) \oplus B
$$

- Morphological closing operation,

$$
A \bullet B=(A \oplus B) \theta B
$$

\section{Hough Circular Transform}

A circle is represented mathematically as $\left(\mathrm{x}^{-}\right.$ $\left.\mathrm{x} \_\{\text {center }\}\right)^{\wedge} 2+\left(\mathrm{y}-\mathrm{y} \_\{\text {center }\}\right)^{\wedge} 2=\mathrm{r}^{\wedge} 2$ where (x_\{center\},y_\{center\}) is the center of the circle, and $\mathrm{r}$ is the radius of the circle. From equation, we can see we have 3 parameters, so we need a 3D accumulator for hough transform, which would be highly ineffective. So OpenCV uses more trickier method, Hough Gradient Method which uses the gradient information of edges.

The function we use here is cv2.HoughCircles(). It has plenty of arguments which are well explained in the documentation. So, we directly go to the code.

\section{Count of Pixel}

In the first paragraph, all pixels of the cv2 image object "img" are extracted and counted with a pixel size of 255 i.e. white pixels. Likewise, the second line says that all "img" image object pixels of cv2 must be extracted and counted, i.e. black pixels.

\section{Simulation Results}

Below Simulation results have been obtained with a Spyder Anaconda. This implementation has shown the robustness, versatility and speed of this smart retinal cancer device. The findings of cancer identification using the training picture package resulted in $100 \%$ detection.

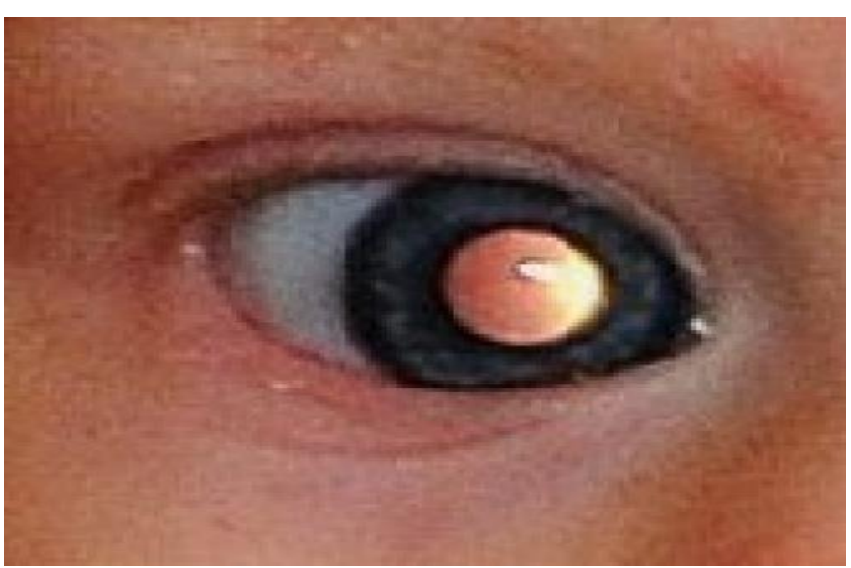

Figure 3: Cancer Image 


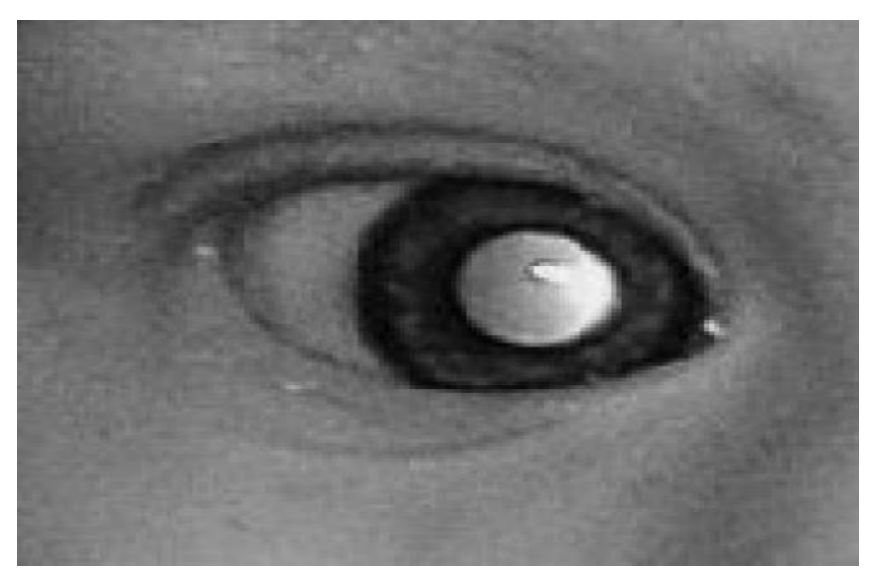

Figure 4: Gray-Scale Image

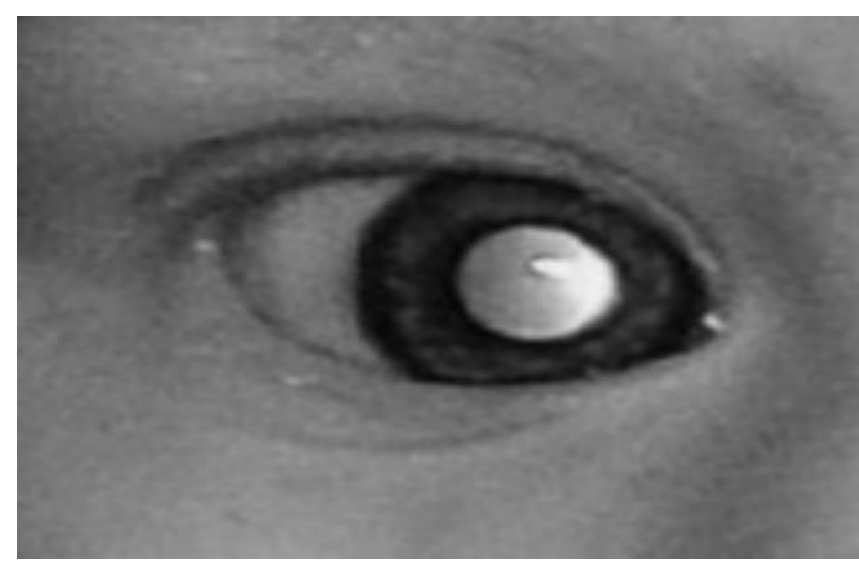

Figure 5: 2-D filtering

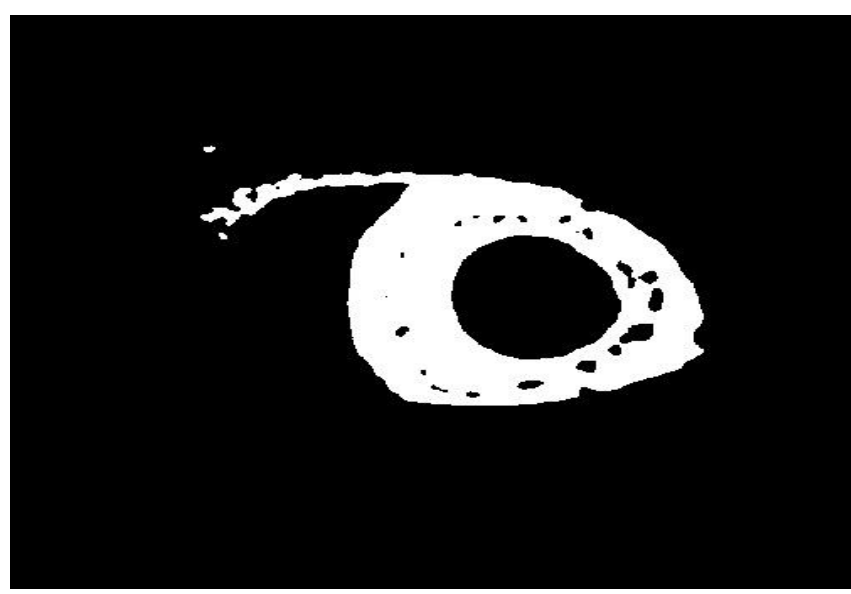

Figure 6: OTSU Thresholding

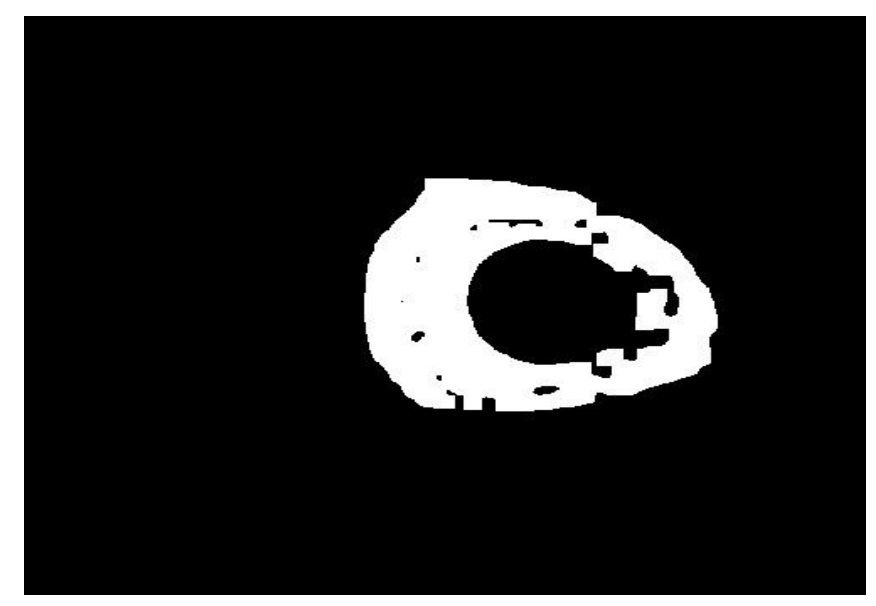

Figure 7: Morphological Output

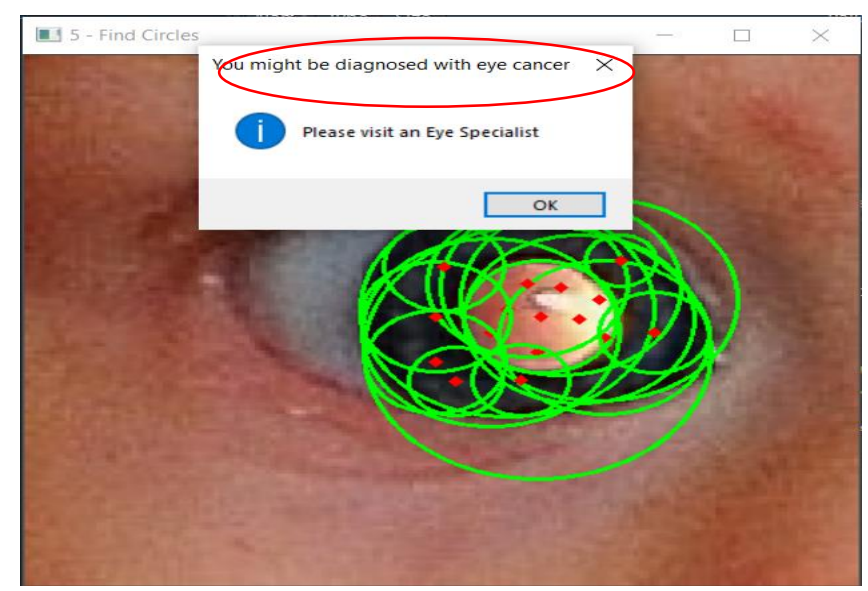

Figure 8: Final Retina cancer Output

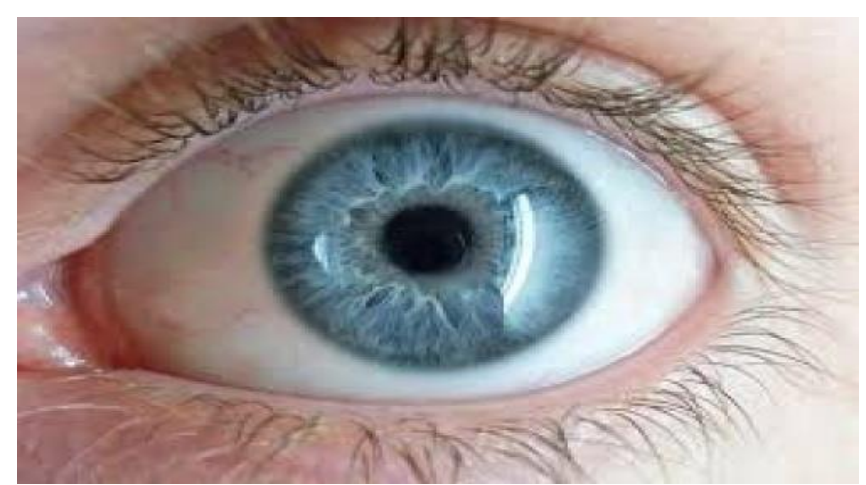

Figure 9: Healthy Retina Image 


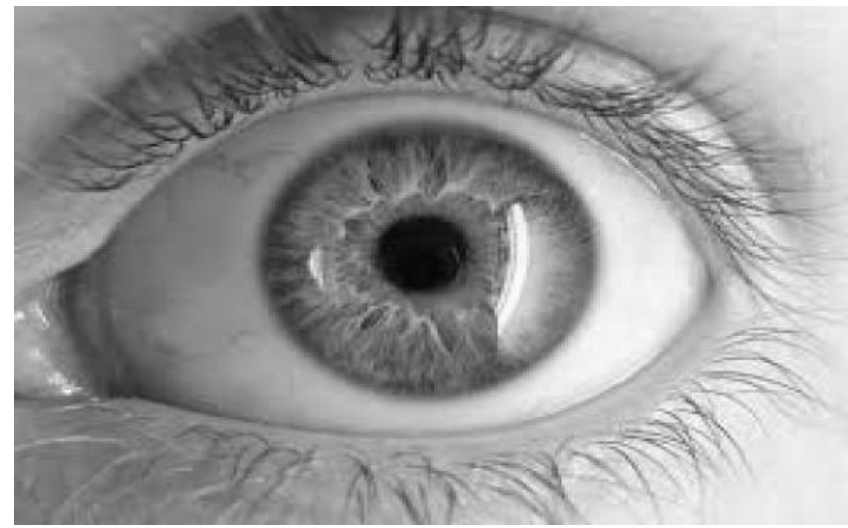

Figure 10: Gray-Scale Image

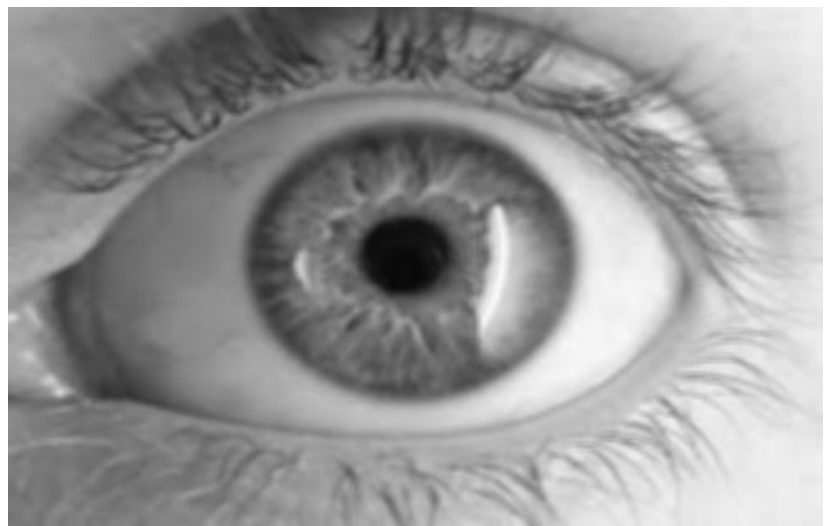

Figure 11: 2-D Filtering

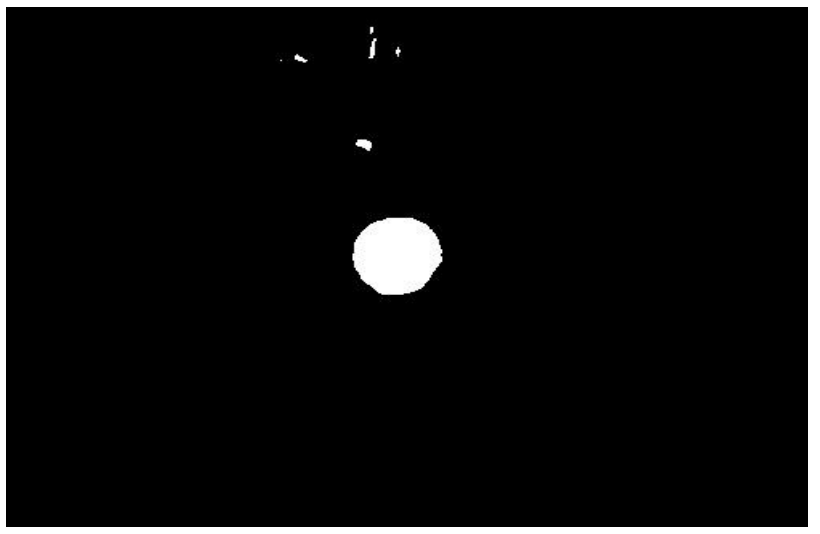

Figure 12: OTSU Thresholding

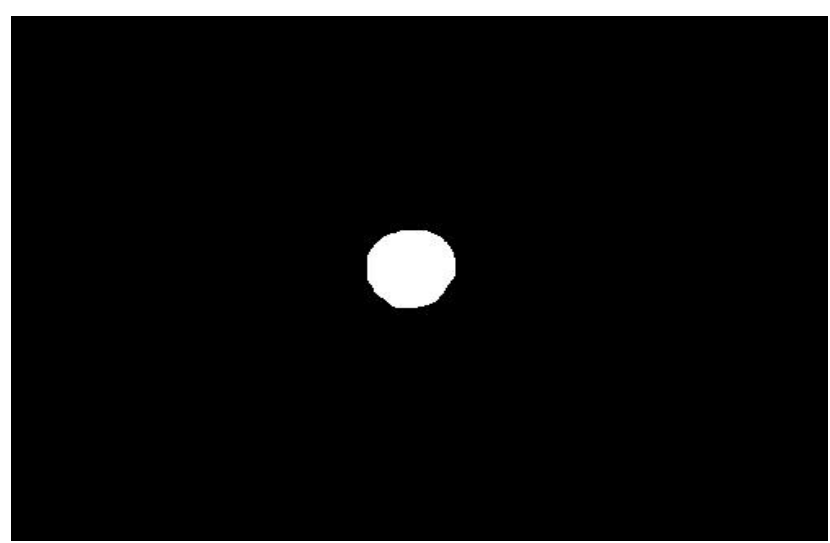

Figure 13: Morphology Output

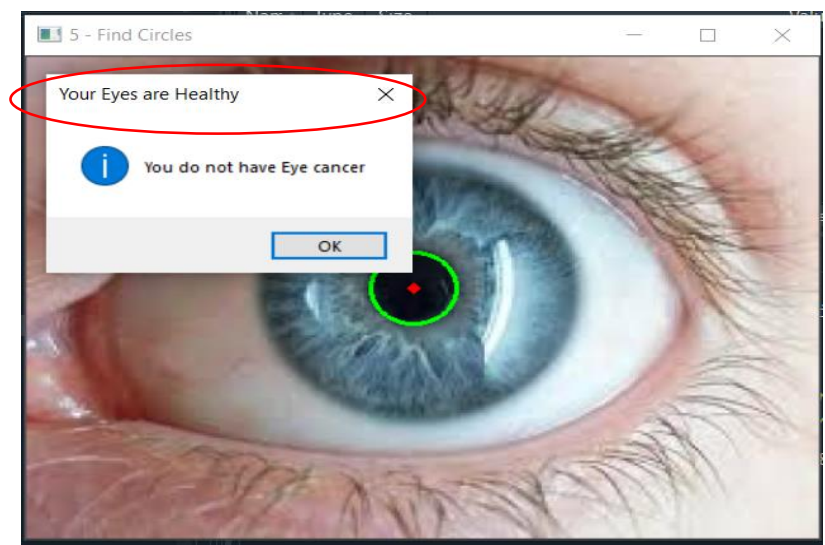

Figure 14: Retina Healthy Output

\section{v. CONCLUSION}

Hough's methods for transforming and threshing retinal cancer have been established in this article. Image processing techniques in medical matters, as in the Lasik machine, are very useful and important. When testing the system, the experimental findings showed that the retinal cancer screening system produced is a robust image treatment that detects all anomalies in the iris region and particularly in small areas. The stability of the system is to note immediately at the first glance some abnormality in the eye. In 0.23 seconds, the system also directly labels the anomalies or cancer in the iris, making our system successful. 


\section{REFERENCES}

[1]. Kamil Dimililer, Yoney Kirsal Ever, Haithm Ratemi, "Intelligent eye Tumour Detection System", Procedia Computer Science, Volume 102, 2016, Pages 325-332, ISSN 1877-0509

[2]. Dimililer K, Kirsal EY, Ugur B. Tumour Detection on CT Lung Images using Image Enhancement, International Science and Technology Conference (ISTEC 2016); ISSN: 2146-7382.

[3]. Lindlahr H. Iris diagnosis and Other Diagnostic Methods. The Lindlahr Publishing Co. Chicago; 1919.

[4]. Dimililer K. Neural network implementation for image compression of $\mathrm{x}$-rays. Electronics World 2012; 118 (1911):26-29.

[5]. World Health Organization. Global Cancer Rates. Available: http://www.who.int/mediacenter/release/2003/p r27/enprinted;2003.Accessed 16 March 2014.

[6]. Grutzmacher RD, Lindquist TD, Chittum ME, Bunt-Milam AH, Kalina RE. Congenital iris cysts. The British journal of ophthalmology 1987; 71 (3): 227-234.

[7]. Dimililer K. Backpropagation neural network implementation for medical image compression. J Appl Math 2013; http://dx.doi.org/10.1155/2013/453098.

[8]. Starr OD, Patel DV, Allen JP, McGhee CN. Iris melanoma: pathology, prognosis and surgical intervention. Clinical \& experimental ophthalmology 2004; $32 \quad$ (3): 294-296. doi:10.1111/j.1442-9071.2004.00821.x.

[9]. Marcus DM, Sahel JA, Jakobiec FA. Pigmented tumors of the iris. In Jakobiec FA. Albert DM (eds) Principles and practice of ophthalmology. W.B. Saunders. Philadelphia; 1994: 3198-3208.

[10]. Albert DM, Kulkarni AD. Intraocular Melanoma. In: DeVita VT, Jr., Lawrence TS, Rosenberg SA (eds) Cancer: Principles and Practice of Oncology. 3rd ed. Lippincott
Williams \& Wilkins. Philadelphia; 2011: 20902098.

[11]. Lye WL,Ali C,Liau CF,Jamal AD. Tumor detection using iris pattern. In Proceeding of the 4th Annual Seminar of National Science Fellowship 2004;363-368.

[12]. Sroubek F, Flusser J. Fusion of Blurred Images. In: Blum R, Liu Z (eds) Multi-Sensor Image Fusion and its Applications, vol 25. Signal Processing and Communications Series 2005;423-449.

[13]. Church JC, Chen Y, Rice SV. A spatial median filter for noise removal in digital images. In: Proceedings of IEEE SoutheastCon, Huntsville, $\begin{array}{lll}\mathrm{AL} & 2008 ; & 618-\end{array}$ doi:10.1109/SECON.2008.4494367.

[14]. Stefanescu R, Pennec X, Ayache N. Grid powered nonlinear image registration with locally adaptive regularization. Med Image Anal 2004; 8 (3): 325-342.

[15]. Eng HL, Ma KK. Noise adaptive soft-switching median filter. IEEE Trans Image Process 2001; 10 (2): 242-251.

\section{Cite this article as :}

Dr. Harsh S Dave, Dr. Vaishnavi Patel, Dr. Aash Gopalak, Dr. Harsh Bhatt, Dr. Sheshang Degadwala, Dhairya Vyas, "Retina Cancer Detection using Thresholding Approach", International Journal of Scientific Research in Science and Technology (IJSRST), Online ISSN : 2395-602X, Print ISSN : 2395-6011, Volume 8 Issue 1, pp. 276-282, JanuaryFebruary 2021. Available at doi : https://doi.org/10.32628/IJSRST207566 Journal URL : https://ijsrst.com/IJSRST207566 